\title{
ANÁLISE DO PADRÃO DE FRATURAS EM PINOS ANATOMIZADOS CIMENTADOS COM DIFERENTES AGENTES CIMENTANTES
}

\author{
Raísa Cristina Madeira Barbosa* \\ Nielsen Barros Souza* \\ Adriana de Fátima Vasconcelos Pereira ${ }^{\text {**x }}$ \\ Vandílson Pinheiro Rodrigues ${ }^{*+* x}$ \\ Ivone Lima Santana
}

RESUMO: 0 objetivo do presente estudo foi investigar, in vitro, o padrão de fratura em dentes com pino de fibra de vidro (PFV) anatomizado e fixado com diferentes agentes cimentantes. Foram utilizados 30 dentes bovinos mandibulares unirradiculares. As raízes foram tratadas endodonticamente e divididas aleatoriamente em dois grupos, de acordo com 0 agente cimentante utilizado, em G1: cimento resinoso autoadesivo (RelyX U200-3M ESPE); G2: cimento resinoso convencional (Allcem-FGM). Após a anatomização e cimentação dos pinos, os espécimes foram armazenados em estufa, a $37^{\circ} \mathrm{C}$, por sete dias. Em seguida, foi realizada a secção das raízes, sob irrigação constante, obtendo-se de cada raiz seis fatias de $1 \mathrm{~mm}$ de espessura, totalizando 180 corpos de prova. 0 teste de resistência à extrusão (push-out) foi realizado em todos os espécimes, e posteriormente, os corpos de prova foram observados em microscópio ótico de luz, para avaliar os tipos de fratura. 0 tipo de fratura mais frequente em ambos os grupos foi a coesina em dentina (G1 $=32,2 \%$; $G 2=30 \%$ ). Observou-se frequência estatisticamente maior de falhas adesivas entre dentina e cimento no grupo G2 $(P=0,007)$. Além disso, detectou-se frequência estatisticamente mais elevada $(P=0,027)$ de fratura coesiva em dentina no terço cervical $(38,3 \%)$ quando comparado ao terço apical $(20,0 \%)$. Os achados sugerem que 0 cimento autoadesivo (RelyX U200-3M ESPE) apresentou resultados mais favoráveis quando da análise do padrão de fraturas.

PALAVRAS-CHAVE: Cimentos dentários; Cimentação; Pinos dentários.

\section{ANALYSIS OF BREAKING PATTERN IN CEMENTED ANATOMIZED PINS WITH DIFFERENT CEMENTING AGENTS}

\begin{abstract}
The breaking pattern in teeth with glass fiber pins (GFP), anatomized and fixed with different cementing agents, is analyzed in vitro. Thirty bovine mandibular uni-radicular teeth were used. Roots were treated endodontically and randomly divided into two groups according to the cementing agent employed, or rather, G1: auto-adhesive resinous cement (RelyX U200-3M ESPE), and G2: conventional resinous cement (Allcem-FGM). After the anatomization and cementation of pins, the specimens were stored in a buffer at $37^{\circ} \mathrm{C}$ for 7 days. Roots were then cut under flowing water and six $1 \mathrm{~mm}$-thick slices were cut, totaling 180 specimens. The push-out test was performed for all specimens and then placed under a light microscope to assess the type of fracture. The most frequent type of fracture for the two groups comprised coesine in dentin (G1 $=32.2 \%$; $G 2=30 \%$ ). There was a statistically greater frequency in adhesive failures between dentin and cement in $G 2(p=0.007)$, with a statistically higher frequency $(p=0.027)$ of cohesive fracture in dentin in the third cervical $(38.3 \%)$ when compared to the third apical (20.0\%). Results suggest that auto-adhesive cement (RelyX U200-3M ESPE) had more positive results in the analysis of fracture patterns.
\end{abstract}

KEYWORDS: Teeth cement; Cementation; Dental pins.

\footnotetext{
* Discente do Curso de Odontologia da Universidade Federal do Maranhão (UFMA), Brasil. E-mail: raisacristina_@hotmail.com

** Doutorando do Programa de Pós-graduação em Odontologia, Instituto Florence de Ensino Superior, Brasil.

${ }^{* * *}$ Docente Adjunta do curso de Odontologia da Universidade Federal do Maranhão (UFMA), Brasil.

${ }^{* * * *}$ Docente Adjunto do curso da Universidade Federal do Maranhão (UFMA), Brasil.

**** Docente Adjunta do curso de Odontologia da Universidade Federal do Maranhão (UFMA), Brasil.
} 


\section{INTRODUÇÃo}

0 tratamento endodôntico, em dentes que apresentam grande comprometimento de sua coroa, viabiliza a utilização de retentores intrarradiculares como meio auxiliar de retenção coronária (SILVEIRA et al., 2011).

Ao longo do tempo, era comum, na prática clínica odontológica, a utilização de retentores metálicos fundidos para reabilitar dentes tratados endodonticamente. Porém, os mesmos apresentam características desfavoráveis. Necessitam de etapa laboratorial, apresentam rigidez superior à dentina $\mathrm{e}$ são passíveis de corrosão. Como agravante tem-se a irregularidade da dissipação das forças mastigatórias e concentração de tensões na região apical, o que pode levar à fratura da raiz (SAUSEN, 2011; GOMEZ-POLO et al., 2010). Tais fatores levaram à busca por retentores constituídos por materiais com propriedades físicas similares à estrutura dentária e com capacidade de adesão a mesma (KAIZER et al., 2009).

Em 1990, Duret et al. introduziram um material não metálico para a fabricação de pinos, com base no princípio de reforço de fibra de carbono. Os pinos originais de fibra de carbono eram escuros, com possibilidade de comprometimento estético. Foram então introduzidos os pinos de fibra de vidro (PFV), de cor branca, a fim de minimizar problemas relativos à questão estética (FERRARI et al., 2000).

Os PFVs permitem uma combinação homogênea dos fatores pino/cimento/dentina dada à adesão que ocorre nesse sistema. Dessa forma, as forças são distribuídas de maneira semelhante àquela que acontece no dente íntegro (FREDRIKSSON et al., 1998).

Um dos fatores para obter o sucesso nas restaurações de dentes com tratamento endodôntico é a retenção do pino no canal radicular. Esta deve resistir às diferentes condições térmicas e mecânicas que estão presentes na cavidade bucal (Ll; WHITE et al., 1999), além de reproduzir anatomicamente 0 endodonto, garantindo dessa forma o íntimo contato do pino com a dentina radicular (SCHWARTZ; FRANSMAN, 2005).

Dentro desse contexto tem-se a anatomização dos PFVs, que consiste no reembasamento de um PFV com resina composta tornando-0 assim apto a se adequar à forma do canal radicular (SOUZA-JÚNIOR et al., 2012).

Além de menor tempo clínico outra vantagem deste método consiste no íntimo contato do pino às paredes do endodonto e dessa forma na redução da quantidade de cimento a ser posicionado no canal. A presença de uma exígua espessura de cimento determina a distribuição mais uniforme das cargas oclusais e permite conter a contração de polimerização e 0 estresse determinado por ela (DE MUNCK et al., 2004).

Para a retenção dos PFVs utilizam-se agentes de cimentação resinosos. Dentre todas as possibilidades têm-se os convencionais, os autoadesivos, os autocondicionantes e os que reúnem as duas situações autoadesivos autocondicionantes (BRAGA et al., 2005).

Os cimentos resinosos autoadesivos são indicados para serem usados sem aplicação de qualquer sistema adesivo prévio. São materiais híbridos que combinam características dos compósitos restauradores, adesivos autocondicionantes e agentes de cimentação (FERRACANE et al., 2010).

Entretanto, alguns fatores restringem $\mathrm{e}$ dificultam a adesão na região radicular. Entre eles podem-se verificar as variações anatômicas próprias da dentina radicular, a formação de smear layer, a provável influência da composição do cimento endodôntico com 0 agente cimentante do pino, a difícil polimerização dos materiais resinosos dentro de uma cavidade extremamente profunda, o tipo de condicionamento ácido que poderia ser incompleto permitindo remanescentes de smear layer além da influência inerente do sistema de pinos (SALAS, 2005).

Dentro desse contexto, além das dificuldades adesivas inerentes à morfologia da dentina radicular, observa-se que ainda não foi estabelecida a força de 
ligação dos PFVs no canal radicular, no que diz respeito aos terços radiculares, quando se utilizam cimentos autoadesivos (FERRARl et al., 2000; ZICARI et al., 2008) e sua influência nas fraturas. Desta forma, 0 estudo da associação entre 0 tipo de agente cimentante e 0 PFV anatomizado pode fornecer maiores informações a respeito da adaptação deste conjunto às paredes do conduto e sua relação com a resistência à fratura $e$ retenção do pino (MUMCU; ERDEMIR; TOPCU, 2010).

Portanto, esta pesquisa tem como objetivo avaliar in vitro 0 padrão de fratura de PFVs anatomizados cimentados com diferentes tipos de agentes cimentantes à dentina radicular.

\section{METODOLOGIA}

\subsection{DELINEAMENTO EXPERIMENTAL}

Realizou-se um estudo experimental, in vitro, no qual foram utilizados 30 dentes, distribuídos aleatoriamente em dois grupos, de acordo com 0 uso dos cimentos resinosos convencional (Allcem) e autoadesivo (RelyX U200) para avaliação do padrão de fratura.

Neste estudo foram utilizadas 30 unidades experimentais as quais foram divididas em dois grupos $(n=15)$. A forma de designar as unidades experimentais a cada grupo ocorreu por randomização. Foram utilizados os cimentos resinosos convencional (Allcem) e autoadesivo (RelyX U200).

\subsection{SELEÇÃO DOS DENTES}

Foram selecionados 30 incisivos bovinos mandibulares hígidos. Os dentes foram devidamente limpos e armazenados em água destilada, sob refrigeração, a fim de prevenir sua desidratação.

Os dentes foram seccionados abaixo da junção amelocementária, de forma perpendicular ao longo eixo do dente, estabelecendo o comprimento radicular de $17 \mathrm{~mm}$. Para a realização deste procedimento, foi utilizado um disco diamantado dupla face $(100 \mathrm{~mm}$ x 0,45 mm x 12,7 mm; ERIOS Equipamentos, São Paulo, Brasil) em máquina de corte de precisão sob abundante irrigação.

\subsection{TRATAMENTO ENDODÔNTICO E DESOBTURAÇÃO DO CONDUTO}

0 tratamento endodôntico foi realizado por meio da técnica clássica manual (COLACO; PAl, 2015), que determina a seleção de um instrumento endodôntico apical inicial seguido de três limas endodônticas de calibre superior, a fim de confeccionar 0 batente apical no comprimento real de trabalho (CRT) estabelecido em $16 \mathrm{~mm}$. 0 irrigante utilizado foi o hipoclorito de sódio a $2,5 \%$.

Para a obturação dos condutos foi realizada a técnica de condensação lateral, utilizando-se cones de guta-percha (Odous De Deus, Belo Horizonte, Brasil) e cimento obturador endodôntico (AH Plus, Dentsply-Maillefer, Ballaigues, Switzerland).

Os espécimes foram armazenados em água destilada a $37^{\circ} \mathrm{C}$ por sete dias, para assegurar a completa presa do cimento endodôntico.

A desobturação do conduto foi realizada com brocas Largo n³ e 4, (Dentsply Ind. Com. Ltda., Petrópolis, RJ, Brasil) deixando $5 \mathrm{~mm}$ de material obturador no ápice.

\subsection{CIMENTAÇÃO DOS PINOS DE FIBRA DE VIDRO E OBTENÇÃO DAS FATIAS RADICULARES}

Foram utilizados 30 pinos de fibra de vidro (PFVs) (WhitePost, FGM, Joinville, Brasil) os quais passaram por um processo de limpeza com álcool $70 \%$ por 1 min, seguido de secagem com jato de ar. Cada PFV foi sinalizado e, em seguida, recebeu uma camada de adesivo (Adper Scotchbond Multiuso Plus, 3M/USA, St. Paul, MN, EUA) que foi então fotoativada por 40s (ULTRALED, $800 \mathrm{~mW} / \mathrm{cm}^{2}$ CuringLight- Dabi Atlante, São Paulo, Brasil). Logo após, a superfície foi coberta por resina composta por Filtek Z350 XT (3M ESPE, St. Paul, Mn, EUA). (Figura 1) 


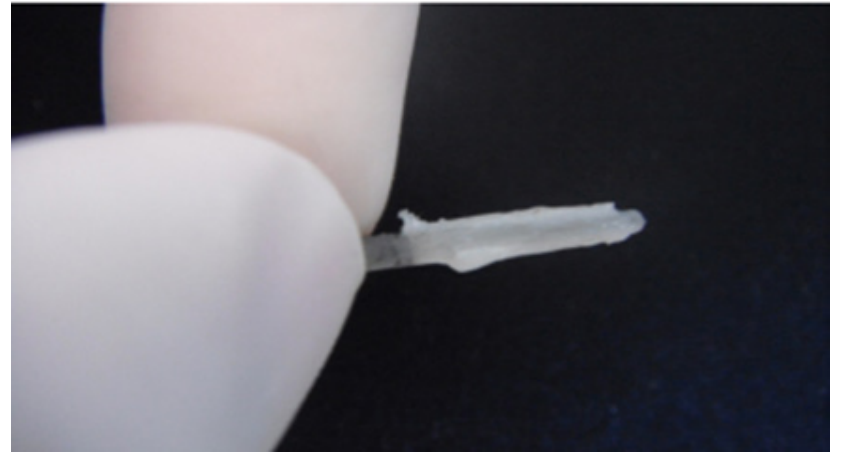

Figura 1. PFV anatomizado com resina composta por Filtek Z350 XT (3M ESPE, St. Paul, Mn, EUA)

0 conduto foi isolado com isolante KY (Johnson \& Johnson, São José dos Campos, SP, Brasil) e então o conjunto pino/resina foi inserido no conduto radicular. Foi feita uma fotoativação de 5 s para início da polimerização, e, após retirada do conjunto pino/ resina do conduto, foi feita nova ativação de $60 \mathrm{~s}$, a fim de polimerizar toda a resina.

Os espécimes foram distribuídos em dois grupos ( $n=15)$ de acordo com o material utilizado na cimentação dos PFVs (Quadro 1). Grupo 1- Cimento Resinoso RelyX U200-3M ESPE; Grupo 2- Cimento Resinoso Allcem- FGM.

Quadro 1. Grupos e técnicas de aplicação dos cimentos resinosos conforme os fabricantes

\begin{tabular}{|c|c|}
\hline Grupo & Técnica de aplicação \\
\hline $\begin{array}{l}\text { Grupo } 1 \\
\text { RelyX U200 - 3M } \\
\text { ESPE }\end{array}$ & $\begin{array}{l}\text { Misturar as duas pastas. Aplicar uma camada } \\
\text { da mistura na superfície do PFV e no interior } \\
\text { do conduto, com auxílio de seringa Centrix. } \\
\text { Posicionar o pino e fotoativar por } 20 \mathrm{~s} \text { ou } \\
\text { aguardar } 6 \text { minutos para a polimerização } \\
\text { química. }\end{array}$ \\
\hline $\begin{array}{l}\text { Grupo } 2 \\
\text { Allcem- FGM }\end{array}$ & $\begin{array}{l}\text { Condicionar a dentina radicular durante } 15 \\
\text { s com gel de ácido fosfórico } 37 \% \text { (CondAc, } \\
\text { FGM). Enxaguar com água, removendo-se } \\
\text { todo } 0 \text { ácido. Secar o conduto com auxílio de } \\
\text { cone de papel absorvente. Aplicar o adesivo } \\
\text { nas paredes do conduto com microaplicador } \\
\text { (Cavibrush, FGM), friccionando-0 no conduto } \\
\text { durante } 15 \mathrm{~s} \text { e fotoativar. Misturar as duas } \\
\text { pastas do Allcem durante } 10 \text { s. Aplicar } \\
\text { uma camada fina e uniforme da mistura na } \\
\text { superfície do PFV, ou no interior do conduto, } \\
\text { com auxílio de seringa Centrix. Posicionar o } \\
\text { PFV no interior do conduto e fotoativar por } \\
40 \text { s. }\end{array}$ \\
\hline
\end{tabular}

Transcorrido o período de sete dias, as raízes foram fixadas em uma base de resina acrílica e seccionadas no sentido transversal, em máquina de corte de precisão, sob irrigação constante, com disco diamantado dupla face. Foram feitas secções obtendo-se seis fatias radiculares referentes aos terços radiculares cervical, médio e apical, com $1 \mathrm{~mm}$ de comprimento cada, totalizando 180 fatias. (Figuras 2 e 3)

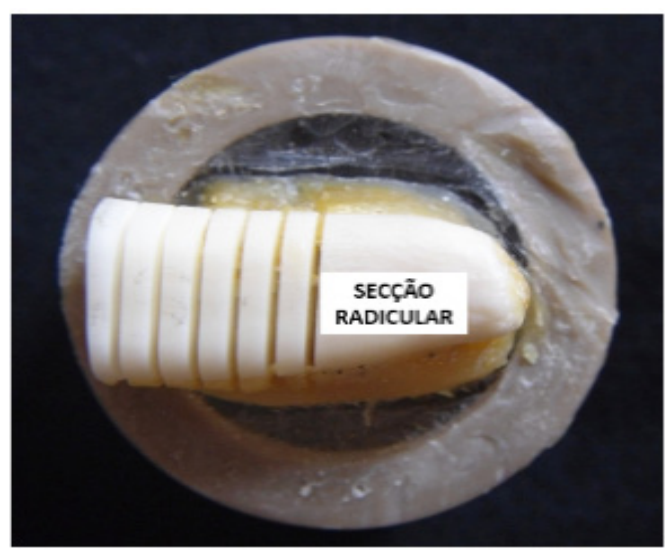

Figura 2. Secção radicular

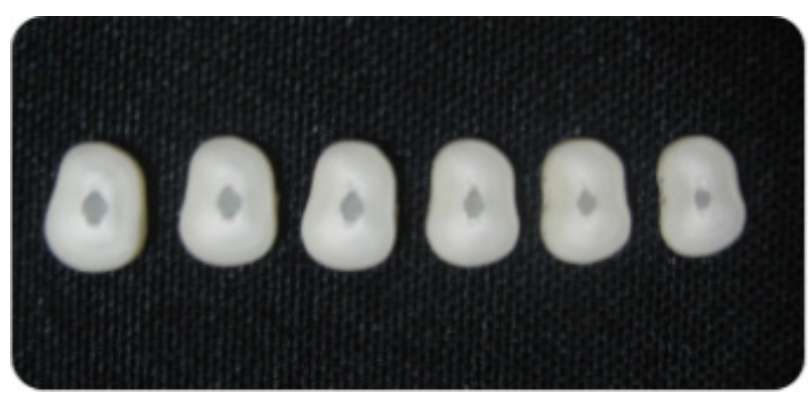

Figura 3. Fatias radiculares

A medição do diâmetro das fatias foi realizada por meio de fotografias das superfícies, superior e inferior de cada uma, usando um Estereomicroscópio (uEye, Alemanha), com ampliação de 30x, capturadas como imagens JPEG. Para cada fatia, tanto 0 diâmetro superior como 0 inferior, foram medidos em pixels, usando o software digital ImageJ (National Institute of Health, Maryland, EUA, http:/rsb.info.nih.gov/ $\mathrm{ij} /$ ). As espessuras das fatias foram medidas com um paquímetro digital, com resolução de $0,01 \mathrm{~mm}$ (Mitutoyo Sul Americana LTDA, Suzano, SP, Brasil). 


\subsection{TESTE PUSH-OUT}

Foi então feito o ensaio de push-out, para as 180 amostras, em uma máquina de ensaio universal (INSTRON ${ }^{\circledR}, 3342$, EUA) a uma velocidade de $0,5 \mathrm{~mm} /$ min, utilizando uma célula de carga de $100 \mathrm{kgf}$, sendo a carga aplicada no sentido ápico-coronal. 0 teste mecânico por extrusão (push-out) pode ser utilizado para medir a resistência adesiva de cimentos resinosos à dentina radicular. Este mede a resistência adesiva ao cisalhamento de fatias transversais dos espécimes, entre dentina e cimento resinoso e cimento resinoso e pino (WANG et al., 2013). Para tal usam-se fatias de $1 \mathrm{~mm}$ de espessura, com carga compressiva, aplicada por meio de uma ponta selecionada, de acordo com 0 diâmetro do pino. A espessura das fatias de $1 \mathrm{~mm}$ permite maior uniformidade na distribuição da carga além de diferenciá-las de acordo com os terços radiculares correspondentes (LATEMPA et al., 2014).

\subsection{ANÁLISE DO PADRÃO DE FRATURA}

Para realizar a análise do padrão de fratura, os espécimes foram levados ao Estereomicroscópio (uEye, Alemanha). Seguiu-se a seguinte classificação: fratura coesiva em dentina; fratura coesiva do pino; falha adesiva na interface cimento/pino; falha adesiva na interface dentina/cimento e falha mista, por meio de análise descritiva.

\subsection{ANÁLISE ESTATÍSTICA}

Os dados foram processados utilizando os recursos do software SPSS, versão 17.0 (IBM, Chicago, IL, USA). A análise estatística incluiu medidas de frequência absoluta e relativa. 0 teste Binomial foi utilizado para comparar as frequências dos tipos de fratura entre os agentes cimentantes e entre os regiões radiculares (cervical versus médio e cervical versus apical). 0 nível de signficância adotado foi de $5 \%$.

\section{RESULTAD0}

A Tabela 1 expressa a distribuição dos tipos de fratura de acordo com 0 agente cimentante utilizado. Após a análise do padrão de fratura, foram verificadas, principalmente, falhas coesivas em dentina em ambos os grupos ( $\mathrm{G} 1=32,2 \%$; $\mathrm{G} 2=30 \%$ ). Observou-se frequência estatisticamente maior de falhas adesivas entre dentina e cimento $(P=0,007)$ no grupo G2 $(16,7 \%)$ quando comparado com o grupo G1 $(4,4 \%)$. As fraturas do tipo coesiva no pino e mista apresentaram baixa frequência na amostra avaliada.

Tabela 1. Distribuição das frequências absoluta e relativa da amostra, segundo o padrão de fratura e agente cimentante

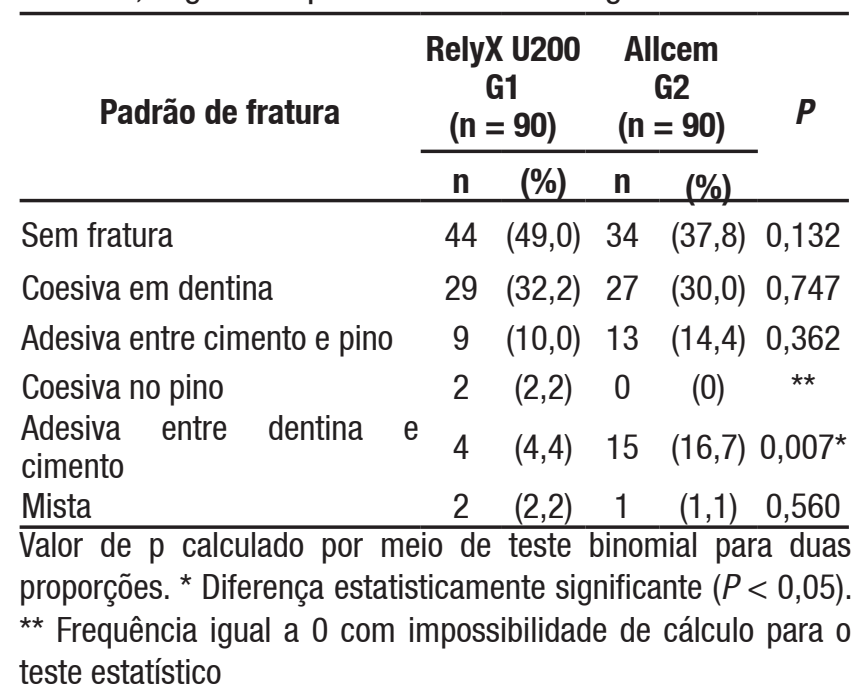

Com relação ao padrão de fratura de acordo com 0 terço radicular avaliado independente do agente cimentante (Tabela 2), detectou-se frequência estatisticamente mais elevada de fratura coesiva em dentina no terço cervical $(38,3 \%)$ quando comparado ao terço apical $(20,0 \%)$. Observou-se que a segunda falha mais frequente na região cervical foi a adesiva entre cimento e pino, enquanto que no terço apical foi a adesiva entre dentina e cimento, o terço médio apresentou frequências semelhantes entre os dois tipos de fratura adesiva. Notou-se também que a fratura coesina no pino ocorreu apenas no terço apical, e não detectou fratura mista neste terço. (Tabela 2). 
Tabela 2. Distribuição das frequências absoluta e relativa da amostra, segundo o padrão de fratura e terço radicular independente 0 agente cimentante

\begin{tabular}{|c|c|c|c|c|c|c|c|c|}
\hline \multirow{3}{*}{ Padrão de fratura } & \multicolumn{6}{|c|}{ Região radicular } & \multirow{3}{*}{$P 1$} & \multirow{3}{*}{$P 2$} \\
\hline & \multicolumn{2}{|c|}{$\begin{array}{l}\text { Cervical } \\
(\mathrm{n}=60)\end{array}$} & \multicolumn{2}{|c|}{$\begin{array}{c}\text { Médio } \\
(\mathrm{n}=60)\end{array}$} & \multicolumn{2}{|c|}{$\begin{array}{c}\text { Apical } \\
(\mathrm{n}=60)\end{array}$} & & \\
\hline & $\mathbf{n}$ & (\%) & $\mathbf{n}$ & (\%) & $\mathbf{n}$ & (\%) & & \\
\hline Sem fratura & 23 & $(38,3)$ & 21 & $(35,0)$ & 31 & $(51,7)$ & 0,704 & 0,142 \\
\hline Coesiva em dentina & 23 & $(38,3)$ & 21 & $(35,0)$ & 12 & $(20,0)$ & 0,704 & $0,027^{*}$ \\
\hline Adesiva entre cimento e pino & 10 & $(16,7)$ & 8 & $(13,3)$ & 6 & $(10,0)$ & 0,609 & 0,282 \\
\hline Coesiva no pino & 0 & (0) & 0 & (0) & 2 & $(3,3)$ & ** & ** \\
\hline Adesiva entre dentina e cimento & 3 & $(5,0)$ & 8 & $(13,3)$ & 9 & $(15,0)$ & 0,113 & 0,067 \\
\hline Misto & 1 & $(1,7)$ & 2 & $(3,4)$ & 0 & (0) & 0,558 & ** \\
\hline
\end{tabular}

Valor de $\mathrm{p}$ calculado por meio de teste binomial para duas proporções. ${ }^{1}$ Análise comparativa da região cervical versus média. ${ }^{2}$ Análise comparativa da região cervical versus apical. * Diferença estatisticamente significante $(P<0,05)$. ${ }^{* *}$ Frequência igual a 0 com impossibilidade de cálculo para o teste estatístico.

\section{DISCUSSÃO}

A odontologia restauradora e estética apresentou crescente desenvolvimento tanto nas técnicas quanto no uso dos diferentes materiais. Observa-se preocupação constante com os fatores de adesão, sendo a adesão do pino ao agente cimentante de fundamental importância (LATEMPA et al., 2014). 0 presente estudo avaliou o padrão de fratura, quando do uso de um agente cimentante convencional e de um cimento autoadesivo.

Pesquisas (CANTOR0 etal., 2011; CASTELLANA et al., 2010; MAZZITELLI et al., 2009) têm sido realizadas utilizando diversos sistemas de cimentos resinosos com o objetivo de verificar a retenção dos pinos à dentina radicular. Porém, os resultados são controversos.

Autores (CANTORO et al., 2011; PEREIRA et al., 2013) informam que 0 uso do cimento resinoso autoadesivo de cura dual sem necessidade de prétratamento do canal radicular, quando da cimentação de um pino de fibra de vidro, apresenta resultados inferiores de adesão quando comparados com 0 sistema adesivo de condicionamento total usado com um cimento resinoso de cura dual. No presente estudo, ao se analisar os padrões de fratura, podese verificar a boa adesão entre dentina e cimento resinoso no grupo G1 (U200), no qual a maioria das falhas encontradas foi coesiva em dentina $(16,1 \%)$. Viotti et al. (2009) verificaram em seu trabalho que a capacidade de ligação de cimentos autoadesivos pode ser atribuída, em parte ou principalmente, à sua capacidade para interagir quimicamente com a hidroxiapatita da dentina, sendo seu mecanismo de união mais químico do que físico.

Nesse estudo, o cimento resinoso convencional (Allcem) apresentou percentual de falhas superior ao do cimento autoadesivo (RelyX U200), quando foram observados os padrões de adesão cimento/ pino e dentina/cimento. Resultados similares foram encontrados por Ferreira et al. (2012) que mencionam também, principalmente, falhas adesivas na interface pino/cimento para grupos cimentados com cimento resinoso convencional. Em contraste, Sarr et al. (2010) encontraram apenas falhas adesivas na interface cimento/dentina para pinos de fibra com e sem reembasamento.

0 fato de 0 G2 (AllCem) ter demonstrado, neste estudo, resultados menos favoráveis, quando verificados os padrões de falhas "adesão dentina/ cimento" e "adesão cimento/pino", pode estar associado à necessidade prévia do cimento quanto ao condicionamento ácido total, envolvendo diversas etapas, o que torna a técnica mais sensível. 
De acordo com Schwartz e Fransman (2005), 0 desenvolvimento dos adesivos autocondicionantes tem como principais vantagens a diminuição no tempo de aplicação, a redução de problemas que possam ocorrer durante a adesão, além de demonstrar ser um protocolo menos sensível e de menor colapso das fibras colágenas por meio da eliminação das etapas de lavagem do ácido e secagem da cavidade, 0 que diminui 0 risco de discrepâncias entre desmineralização e penetração do monômero na dentina. Estes fatores também ajudam a explicar os percentuais mais favoráveis no grupo G1 (RelyX U200) observados neste estudo, quanto às falhas adesivas em "dentina/cimento" e "cimento/pino".

A principal característica do cimento resinoso autoadesivo é a capacidade de adesão às estruturas dentais sem condicionamento prévio ou aplicação de adesivo. Portanto, sua técnica de aplicação estabelece apenas um passo clínico, 0 que 0 torna muito atrativo do ponto de vista clínico. É necessário apenas secar o remanescente dentário e proceder a etapa de cimentação. Esta facilidade de execução tem feito dos cimentos autoadesivos os mais utilizados na cimentação de pinos de fibra de vidro, atualmente (WEISER et al., 2015).

Com relação aos terços radiculares, verificase que a qualidade da adesão também pode ser influenciada pela conformação da dentina, uma vez que ocorre a diminuição da densidade e do diâmetro dos túbulos dentinários em direção apical. Este fator pode influenciar no mecanismo de ligação micromecânica ou química dos materiais utilizados (ZHANG et al., 2008). No presente estudo, quando foram analisados os padrões de fratura "coesiva em dentina" e "adesiva entre cimento e pino", observou-se que ocorreu diminuição no número de falhas no sentido do terço cervical para o terço apical.

Quando da análise dos padrões de fratura "coesiva no pino" e "adesiva entre dentina e cimento", foi observada maior frequência de falhas no terço apical, quando comparado com os terços cervical e médio. Estes resultados corroboram com outros estudos que informam o fato de as falhas na interface adesiva pino/cimento estarem relacionadas a fatores como dificuldade de acesso à região apical, limitações de escoamento do cimento e decrescente efetividade da fotopolimerização nas regiões mais distantes da fonte de luz (DURÃO et al. 2007).

\section{CONCLUSÃO}

Os achados deste estudo sugerem que 0 tipo de agente cimentante pode influenciar o padrão de fratura de pinos anatomizado utilizados em dentes tratados endodonticamente, apresentando 0 cimento autoadesivo RelyX U200 resultados mais favoráveis. Além disso, o terço radicular representa um fator que interfere na adesão. No terço cervical ocorreu fratura do tipo coesiva em dentina com maior frequência que no terço apical, enquanto que a fratura do tipo adesiva entre dentina e cimento ocorreu com maior frequência no terço apical, sugerindo maior fragilidade adesiva nesta última região.

\section{REFERÊNCIAS}

BRAGA, R.R.; BALLESTER, R.Y.; FERRACANE, J.L. Factors involved in the development of polymerization shrinkage stress in resin-composites: a systematic review. Dental Materials, v. 21, n. 10, p. 962-970, abr. 2005.

CANTORO, A. et al. Retentive strength and sealing ability of new self-adhesive resin cements in fiber post luting. Dental Matererials, v. 27, n.10, p. 197204, out. 2011.

CASTELLANA, C.S. et al. Measuring bond strength between fiber post and root dentin: a comparison of different tests. The Journal of Adhesive Dentistry, v. 12. n. 6, p. 477-485, set. 2010.

COLACO, A.S.; PAI, V.A. Comparative Evaluation of the Efficiency of Manual and Rotary Gutta-percha Removal Techniques. American Association of Endodontists, v. 41. n. 11, p. 1871-1874, nov. 2015.

DE MUNCK, J. et al. Bonding of an autoadhesive luting material to enamel and dentin. Dental Matererials, v. 